\title{
The monodromy in the Hamiltonian Hopf bifurcation
}

\author{
J.J. Duistermaat
}

\begin{abstract}
A simple, straightforward computation is given of the monodromy near an equilibrium point of a Hamiltonian system with two degrees of freedom, which is close to a nondiagonalizable resonance.
\end{abstract}

\section{Introduction}

A Hamiltonian Hopf bifurcation is a smooth Hamiltonian system of two degrees of freedom with an equilibrium point and which depends smoothly on a parameter, such that for the special value of the parameter the linear part of the vector field at the equilibrium point has purely imaginary eigenvalues and is not diagonalizable. We use the extensive discussion of van der Meer [5] as our background reference.

Let $x_{1}, x_{2}, y_{1}, y_{2}$ be a standard symplectic coordinate system, the origin of which is taken as the equilibrium point of the Hamiltonian system. As in [5, p. 57], we introduce the functions $X, Y, S$ defined by

$$
X=\frac{1}{2}\left(x_{1}^{2}+x_{2}^{2}\right), \quad Y=\frac{1}{2}\left(y_{1}^{2}+y_{2}^{2}\right), \quad S=x_{1} y_{2}-x_{2} y_{1} .
$$

The Hamiltonian vector field $H_{S}$ of $S$ is equal to the semisimple part of the linear part of the Hamiltonian vector field at the origin. The $\mathrm{H}_{S}$-flow, also referred to as the $S$-action, consists of the synchronous standard rotations in the $\left(x_{1}, x_{2}\right)$-plane and the $\left(y_{1}, y_{2}\right)$-plane.

The first step in obtaining a normal form consists of the observation that there exists an $S$-equivariant formal symplectic change of coordinates which turns the Taylor expansion of the Hamiltonian function so that it is $S$-invariant, up to any desired order, see [5, Ch. 2]. This implies that, up to any order, the Hamiltonian system near the origin behaves as in integrable one, with the function $S$ as the second integral of motion. In the sequel we will restrict the discussion to the case that the Hamiltonian function $H$ is $S$-invariant .

For the terms up to order four the Hamiltonian function $H$ is given by [5, (3.26)], for some constants $\alpha, a_{1}, a_{2}, a_{3}$, where $\alpha \neq 0$. According to [5, Cor. 3.39, p.64], if one has the nondegeneracy condition that $a_{2} \neq 0$ and the linear part of the Hamiltonian vector field moves in a generic way through the resonance, then near the origin the $(S, H)$-fibration is diffeomorphic to the $(S, G)$-fibration, when

$$
G=X+\nu Y+a Y^{2} .
$$


Here $a= \pm 1$ and is equal to the sign of $a_{2}$, and $\nu$ is diffeomorphic to the parameter on which the system depends. The system passes through the resonance when $\nu$ passes through zero. The normal form only holds for small values of $|\nu|$. For $\nu>0$ the linear part has two distinct pairs of purely imaginary eigenvalues whereas for $\nu<0$ two of the eigenvalues have positive real parts and the other eigenvalues are their opposites. In other words, for $\nu>0$ and $\nu<0$ the equilibrium point is elliptic and hyperbolic, respectively.

Only for $\nu<0$ the origin in the $(S, G)$-plane is an isolated singular value, cf. [5, Fig. 4.16, 17 on p. 79]. For $(s, g) \in \mathbf{R}^{2}$, write $F_{(s, g)}$ for the set of $(x, y) \in \mathbf{R}^{4}$ such that $S(x, y)=s$ and $G(x, y)=g$. That is, $F_{(s, g)}$ is the fiber of the mapping $(S, G)$ for the value $(s, g)$. From $[5$, Table 4.1, p.80] we read off that if $a=-1$, then the regular fibers are cylinders, extending out of the neighborhood $U$ of the origin in which the $(S, G)$-fibration is diffeomorphic to the $(S, H)$-fibration. Actually, in this case the non-equilibrium solutions of the Hamiltonian system leave $U$ both for increasing and for decreasing time, so there is no interesting local dynamics.

For this reason we restrict ourselves from now on to the case $\nu<0, a=1$. In this case the origin in the image plane of the mapping $(S, G)$ is an isolated singular value and the fibers $F_{(s, g)}$ for the regular values $(s, g)$ of $(S, G)$ are two-dimensional tori, cf. [5, Table 4.1, p.80]. Combining the equation $g=X+\nu Y+Y^{2}$ with the inequalities $X \geq 0, Y \geq 0$, we see that $2 Y \leq-\nu+\sqrt{\nu^{2}+4 g}, X \leq g+\nu^{2} / 4$, which implies that the fibers $F_{(s, g)}$ are close to the origin if $g$ and $\nu$ are close to zero.

Because the regular fibers $F_{(s, g)}$ of $(S, G)$ are two-dimensional tori, their fundamental groups $\Lambda_{(s, g)}$ are isomorphic to their first homology groups and to the two-dimensional lattice $\mathbf{Z}^{2}$. The isomorphism of $\Lambda_{(s, g)}$ with $\mathbf{Z}^{2}$ depends on the choice of a $\mathbf{Z}$-basis in $\Lambda_{(s, g)}$. The dependence of $\Lambda_{(s, g)}$ on the regular values $(s, g)$ is smooth. This means that the $\Lambda_{(s, g)}$ together form a smooth manifold $\Lambda$, in such a way that the projection $\rho$, which maps each $\lambda \in \Lambda_{(s, g)}$ to $(s, g)$, is a local diffeomorphism from $\Lambda$ onto the set $R$ of regular values. If $\gamma$ is a closed curve in $R$ around the origin, starting and ending at the point $(s, g) \in R$, then the lift of $\gamma$ in $\Lambda$ which starts at $\lambda \in \Lambda_{(s, g)}$ will end at an element $M_{(s, g)}(\lambda) \in \Lambda_{(s, g)}$. This defines an automorphism $M_{(s, g)}$ of $\Lambda_{(s, g)}$, which is called the monodromy of the $(S, G)$-fibration. A Z Z-basis in $\Lambda(s, g)$ can be chosen so that it depends continuously (hence smoothly) on $(s, g)$, if and only if the monodromy is trivial, that is $M_{(s, g)}$ is equal to the identity in $\Lambda_{(s, g)}$. For a discussion of nontriviality of the monodromy as the first obstruction to the existence of global action-angle coordinates for the integrable Hamiltonian system, see [4].

For the first basis vector $c_{1}(s, g)$ in $\Lambda_{(s, g)}$ it is natural to choose the equivalence class of an $S$-circle in $F_{(s, g)}$. Clearly $c_{1}$ is a globally defined smooth section of the projection $\rho: \Lambda \rightarrow R$, hence $c_{1}(s, g)$ is fixed by $M_{(s, g)}$. Furthermore, because the $G$-action commutes with the $S$-action, the $G$-action induces an action in the quotient of the phase space by the $S$-action, which is called the reduced $G$-action. The solutions of the reduced $G$-action are periodic. These sweep out circles which are the quotients of the fibers $F_{(s, g)}$ by the $S$-circle action. The quotient groups $\Lambda_{(s, g)}^{\prime}:=\Lambda_{(s, g)} / \mathbf{Z} c_{1}(s, g)$ form a manifold $\Lambda^{\prime}$ with projection $\rho^{\prime}: \Lambda^{\prime} \rightarrow R$. For each $(s, g) \in R$, the solution of the reduced $G$-system, during one period, defines a $\mathbf{Z}$-basis element $c_{2}^{\prime}(s, g)$ of $\Lambda_{(s, g)}^{\prime}$. Together these define a global section $c_{2}^{\prime}$ of $\rho^{\prime}$. If $c_{2}(s, g) \in \Lambda_{(s, g)}$ is chosen such that $c_{2}(s, g)+\mathbf{Z} c_{1}(s, g)=c_{2}^{\prime}(s, g)$, then $c_{1}(s, g)$ and 
$c_{2}(s, g)$ form a $\mathbf{Z}$-basis of $\Lambda_{(s, g)}$. Moreover, the monodromy $M(s, g)$ acts trivially on $\Lambda_{(s, g)}^{\prime}$, which means that there exists an integer $p$ such that $M_{(s, g)} c_{2}(s, g)=c_{2}(s, g)+p \cdot c_{1}(s, g)$. It is easy to see that the number $p$ neither depends on the choice of the element $c_{2}(s, g) \in$ $c_{2}^{\prime}(s, g)$ nor on the choice of the point $(s, g)$ in the connected set $R$ of regular values. The number $p$ will be called the shift number of the monodromy. It is equal to zero if and only if the monodromy is trivial. We will prove:

For the $(S, G)$-fibration with $a=1$ and $\nu<0$, the shift number of the monodromy is equal to -1 .

In [5, Cor. 4.14, p. 83] it had been concluded that the monodromy is nontrivial, by using an argument of Cushman, based on the fact that the topology of $G^{-1}(\{g\})$ changes if $g$ passes through the origin. See [4] and [1]. Cushman and van der Meer [3] observed that the Hamiltonian Hopf bifurcation occurs in the Lagrange top. The determination of Cushman and Knörrer [2] of the monodromy in the Lagrange top implies that the shift number of the monodromy in the Hamiltonian Hopf bifurcation must be equal to \pm 1 . However, all these monodromy computations refer back to [4] or [1], which either use somewhat elaborate computations of period integrals or the change of the topology in the energy hypersurfaces.

It is the purpose of this paper to show that the monodromy of the Hamiltonian Hopf bifurcation can be determined in a very simple, straightforward way.

\section{Computation of the shift number}

If $y_{1}=y_{2}=0$, then $S=0$ and $G=X \geq 0$. Let $R_{0}$ be the set of $(s, g) \in R$ such that not $s=0$ and $g \geq 0$. Let $C=\rho^{-1}\left(R_{0}\right)$ and let $\pi$ be the projection $(x, y) \mapsto y$. With these notations, the observation that $S=0$ and $G \geq 0$ when $y=0$ just means that $0 \notin \pi(C)$.

The projection $\pi$ conjugates the $S$-action to the standard rotation in the $y$-plane minus the origin, to which any ray is a global section. We will choose the ray $A$ which consists of the $y \in \mathbf{R}^{2}$ such that $y_{1}=0$ and $y_{2}>0$. It follows that the hypersurface $B:=C \cap \pi^{-1}(A)$, the set of $(x, y) \in C$ such that $y_{1}=0, y_{2}>0$, is a global section of the $S$-action in $C$, the set of $(x, y) \in \mathbf{R}^{4}$ such that $(S(x, y), G(x, y)) \in R_{0}$.

In particular, for each $(s, g) \in R_{0}$ the set $F_{(s, g)} \cap B$ is a global section of the $S$-action in $F_{(s, g)}$. The projection along the $S$-orbits maps $F_{(s, g)}$ to a circle. Therefore $F_{(s, g)} \cap B$ is a circle in $F_{(s, g)}$ which intersects the $S$-circle once, so the class of $F_{(s, g)} \cap B$, providided with the correct orientation, can be taken as the second basis element $c_{2}(s, g)$ in $\Lambda_{(s, g)}$. If these $c_{2}(s, g),(s, g) \in R_{0}$, would extend to a continuous function of $(s, g) \in R$, then the monodromy would be trivial. However, the curves $F_{(s, g)} \cap B$ have a singular behaviour when $(s, g)$ converges to a point of $R \backslash R_{0}$. We will determine the shift number of the monodromy from an analysis of this singular limit. We begin with a closer look at the regular case when $(s, g) \in R_{0}$.

If in $B$ we fix the level $s$ of $S=x_{1} y_{2}$, then the resulting manifold $B \cap S^{-1}(\{s\})$ is parametrized by $x_{2}$ and $y_{2}$. In these coordinates the substitution of $x_{1}=s / y_{2}$ leads to the 
formula

$$
G_{s}\left(x_{2}, y_{2}\right):=\frac{1}{2} x_{2}^{2}+V_{s}\left(y_{2}\right), \quad y_{2}>0
$$

for $G$, where

$$
V_{s}\left(y_{2}\right):=\frac{1}{2} f_{s}\left(y_{2}^{2}\right), \quad f_{s}(\tau):=s^{2} / \tau+\nu \tau+\tau^{2} / 2
$$

Because $d^{2} f_{s}(\tau) / d \tau^{2}=2 s^{2} / \tau^{3}+1$ is positive for $\tau>0, d f_{s}(\tau) / d \tau$ has precisely one positive zero $\tau$, even if $s=0$. Consequently, the function $V_{s}$ has precisely one minimum on the positive real axis at a point $y_{2}=y_{2}(s)$ which depends smoothly on $s$, and $d V_{s}\left(y_{2}\right) / d y_{2}<$ 0 if $0<y_{2}<y_{2}(s), d V_{s}\left(y_{2}\right) / d y_{2}>0$ if $y_{2}>y_{2}(s)$. This in turn implies that the level sets of the $(S, G)$-mapping in $B$ are diffeomorphic to circles. Combining this with the $S$-action, we recover the result of $[5$, Table 4.1, p.80] that if $a=1$ and $\nu<0$, then the regular level sets of the map $(S, G)$ are two-dimensional tori.

If we identify the space of $S$-circles in $S^{-1}(\{s\})$ with $S^{-1}(\{s\}) \cap B$, parametrized by $\left(x_{2}, y_{2}\right), y_{2}>0$, then the reduced $\mathrm{H}_{G}$-system becomes identified with the Hamiltonian system in the upper $\left(x_{2}, y_{2}\right)$-plane, defined by the function $G_{s}$. We may think of $G_{s}$ as kinetic energy $x_{2}^{2} / 2$ plus potential energy $V_{s}\left(y_{2}\right)$, except that $d y_{2} / d t=-x_{2}$, so the time variable has the wrong orientation for this interpretation. Because $d x_{2} / d t=d V_{s}\left(y_{2}\right) / d y_{2}$, the orientation of the solutions is determined by the condition that $d x_{2} / d t$ is negative when $y_{2}$ is minimal along the orbit and positive when $y_{2}$ is maximal. Because $x_{1}=s / y_{2}$, for $s \neq 0$ this corresponds to the condition that $d x_{2} / d t<0$ when $\left|x_{1}\right|$ is minimal and $d x_{2} / d t>0$ when $\left|x_{1}\right|$ is maximal along the orbit. (Note that $s \neq 0$ implies that $x_{1} \neq 0$.)

If $(s, g) \in R_{0}$, then the class of the $G_{s}$-solution curve $\gamma_{(s, g)}$, embedded into $B$ by means of the substitutions $x_{1}=s / y_{2}, y_{1}=0$, and followed for one period, will be taken as the second generator $c_{2}(g, s)$ of $\Lambda_{(s, g)}$. The complement of $R_{0}$ in $R$ consists of the points $\left(0, g_{0}\right)$ such that $g_{0} \geq 0$. Let $g_{0}>0$ be fixed. In order to determine the shift number of the monodromy, we investigate the limit behaviour of $\gamma_{(s, g)}$, when $s \uparrow 0, g \rightarrow g_{0}$ and when $s \downarrow 0, g \rightarrow g_{0}$.

The limit set of the orbits for $s<0$ is contained in the set of points $(x, y)$ where $y_{1}=0, y_{2} \geq 0, x_{1} y_{2}=S=0$ and $X+\nu Y+Y^{2}=g_{0}$. There is a piece $x_{1}=0$, which is parametrized by the curve $\delta$

$$
\frac{1}{2} x_{2}^{2}+\frac{1}{2} \nu y_{2}^{2}+\frac{1}{4} y_{2}^{4}=G_{0}\left(x_{2}, y_{2}\right)=g_{0}, \quad y_{2} \geq 0
$$

in the closed upper half of the $\left(x_{2}, y_{2}\right)$-plane. And there is another piece $y_{2}=0$, which in view of the equation $g_{0}=G=X$ is parametrized by the semicircle $\epsilon_{-}$of radius $\sqrt{2 g_{0}}$ in the closed left half of the $\left(x_{1}, x_{2}\right)$-plane. Note that in $B$ the coordinate $x_{1}=s / y_{2}$ has the same sign as $s$, which explains why $x_{1} \leq 0$ at limit points for $s \uparrow 0$.

The curve $\delta$ has to be run through in such a way that $d x_{2} / d t>0$ when $y_{2}$ is maximal, in which case $x_{2}=0$. It follows that we have to come from the singular limit point where $y_{2}=0$ and $x_{2}<0$. Then, while $x_{2}$ remains negative, $y_{2}$ grows to its maximum value, where $x_{2}$ passes zero, after which $x_{2}$ remains positive and $y_{2}$ diminishes to zero, the other singular limit point.

The semicircle $\epsilon_{-}$is also approached by $\gamma_{(s, g)}$, namely the part which corresponds to the $G_{s}$-curve which for $s$ close to zero has small values of $y_{2}$. Here the speed $d x_{2} / d t$ becomes 
very large negative, so we have to slow down the motion to get a continuous curve as a limit, which is the semicircle $\epsilon_{-}$, run through in the counterclockwise direction. Note that the slowing down can be realized by a homotopy of curves. It follows that the class of the resulting loop, consisting of the curve $\delta$, followed by $\epsilon_{-}$run through in the counterclockwise direction, is equal to the limit of $c_{2}(s, g)$ for $s \uparrow 0$ and $g \rightarrow g_{0}$.

Let $\epsilon_{+}$be the semicircle of radius $\sqrt{2 g_{0}}$ in the closed left half of the $\left(x_{1}, x_{2}\right)$-plane. In a similar way we get that the class of the loop, consisting of the curve $\delta$, followed by $\epsilon_{+}$run through in the clockwise direction, is equal to the limit of $c_{2}(s, g)$ for $s \downarrow 0$ and $g \rightarrow g_{0}$. If we substract the previous limit from this one, we get on the one hand the circle in the $x$-plane, run through in the clockwise direction, and on the other hand the increase in $c_{2}(g, s)$ as $(g, s)$ runs around the origin in the counterclockwise direction. Because the class of the circle in the $x$-plane, with $y=0$, run through in the clockwise direction, is

equal to $-c_{1}$, we have completed the proof that the shift number of the monodromy is equal to -1 .

\section{References}

[1] R. Cushman: Geometry of the energy momentum mapping of the spherical pendulum. C.W.I. Newsletter 1 (1983) 4-18.

[2] R. Cushman and H. Knörrer: The energy momentum mapping of the Lagrange top. pp. 12-24 in: Differential Geometric Methods in Mathematical Physics (Proceedings, Clausthal 1983). Eds.: H.D. Doebner and J.D. Hennig. Lecture Notes in Mathematics 1139. Springer-Verlag, Berlin, etc., 1985.

[3] R. Cushman and J.C. van der Meer: The Hamiltonian Hopf bifurcation in the Lagrange top. pp. 26-38 in: Géométrie Symplectique et Mécanique (Proceedings, La Grande Motte 1988). Ed.: C. Albert. Lecture Notes in Mathematics 1416. SpringerVerlag, berlin, etc., 1990.

[4] J..J. Duistermaat: On global action-angle coordinates. Comm. Pure Appl. Math. 33 (1980) 687-706.

[5] J.-C. van der Meer: The Hamiltonian Hopf Bifurcation. Lecture Notes in Mathematics 1160, Springer-Verlag, Berlin etc., 1985.

Mathematisch Instituut, Postbus 80010, 3508 TA Utrecht, The Netherlands. e-mail: duis@math.ruu.nl 